\title{
A noninvasive diagnostic tool to differentiate myocarditis from myocardial infarction: late gadolinium enhanced cardiac magnetic resonance
}

\author{
Yvan Mivelaz • Nicole Sekarski • Salah Dine Qanadli • \\ Erik Jan Meijboom • Stefano Di Bernardo
}

Received: 31 July 2006 /Revised: 28 September 2006 / Accepted: 28 September 2006 / Published online: 7 November 2006

(C) Springer-Verlag 2006

\begin{abstract}
Studies in adults have shown that late gadolinium enhanced cardiac magnetic resonance is a safe and noninvasive diagnostic tool which allows one to differentiate myocardial infarction from myocarditis. We believe that it may also be highly useful in the paediatric population for the same purpose.
\end{abstract}

Keywords Magnetic resonance imaging · Myocardial infarction · Myocarditis · Cardiomyopathy $\cdot$ Children

\section{Case report}

An 11-year-old boy was brought to the emergency department 2 hours after waking with crushing retrosternal chest pain irradiating to the upper left arm and the neck. His only cardiovascular risk factor was a family history of familial hypercholesterolemia and early coronary artery disease (grandfather had his first myocardial infarction at 34 years, father was diagnosed as having silent myocardial infarction and was followed since the age of 38).

He was hemodynamically in a stable condition. Cardiovascular examination was unremarkable except for mild sinus tachycardia. A 12 lead ECG demonstrated a 2 mm ST

Y. Mivelaz $(\bowtie) \cdot$ N. Sekarski $\cdot$ E. J. Meijboom • S. Di Bernardo Pediatric Cardiology, Cardiovascular and Metabolic Diseases Center, Lausanne University Hospital (CHUV),

Rue du Bugnon 46,

Lausanne 1011, Switzerland

e-mail: Yvan.Mivelaz@chuv.ch

S. D. Qanadli

Radiology, Cardiovascular and Metabolic Diseases Center, Lausanne University Hospital (CHUV),

Lausanne, Switzerland segment elevation in leads V5, V6 and $1 \mathrm{~mm}$ in leads II and aVF. Cardiac enzymes were elevated (creatine kinase $1075 \mathrm{U} / \mathrm{L}$ [normal <190], creatine kinase-MB 17\% [normal $<6 \%$ total creatine kinase], troponin I $20 \mathrm{mcg} / \mathrm{L}$ [normal $<0.04 \mathrm{mcg} / \mathrm{L}])$.

Transthoracic echocardiography suggested slight left ventricular hypokinesia. Cardiac catheterization was performed to exclude a coronary artery disease. Coronarography was unremarkable and left ventricular angiography confirmed global hypokinesia. The child underwent cardiac magnetic resonance (CMR) for suspicion of myocarditis. Cine imaging sequences did not reveal focal wall thinning nor wall motion abnormalities. However, late gadolinium enhanced CMR revealed subepicardial enhancement in the inferior-lateral wall of the left ventricle (Fig. 1a,b).

The findings being consistent with myocarditis, the child was placed on steroids leading to fast resolution of symptoms. Normalization of creatine kinase, troponin I, ECG and echocardiography occurred over the following days.

\section{Discussion}

Myocarditis is an inflammatory process resulting in necrosis and/or degeneration of adjacent myocytes not typical of ischemic damage. In the presence of coronary artery disease, coronary blood flow may be reduced and typically induces a focal lesion starting in the subendocardial layer of the myocardium in a coronary artery territory. In myocarditis on the other hand, the histologic changes vary widely; mid-myocardial to epicardial patchy lesions are more commonly observed [1].

Prior to CMR, the "gold standard" for the diagnosis of myocarditis was endomyocardial biopsy because histopathological examination was the only way to show the 

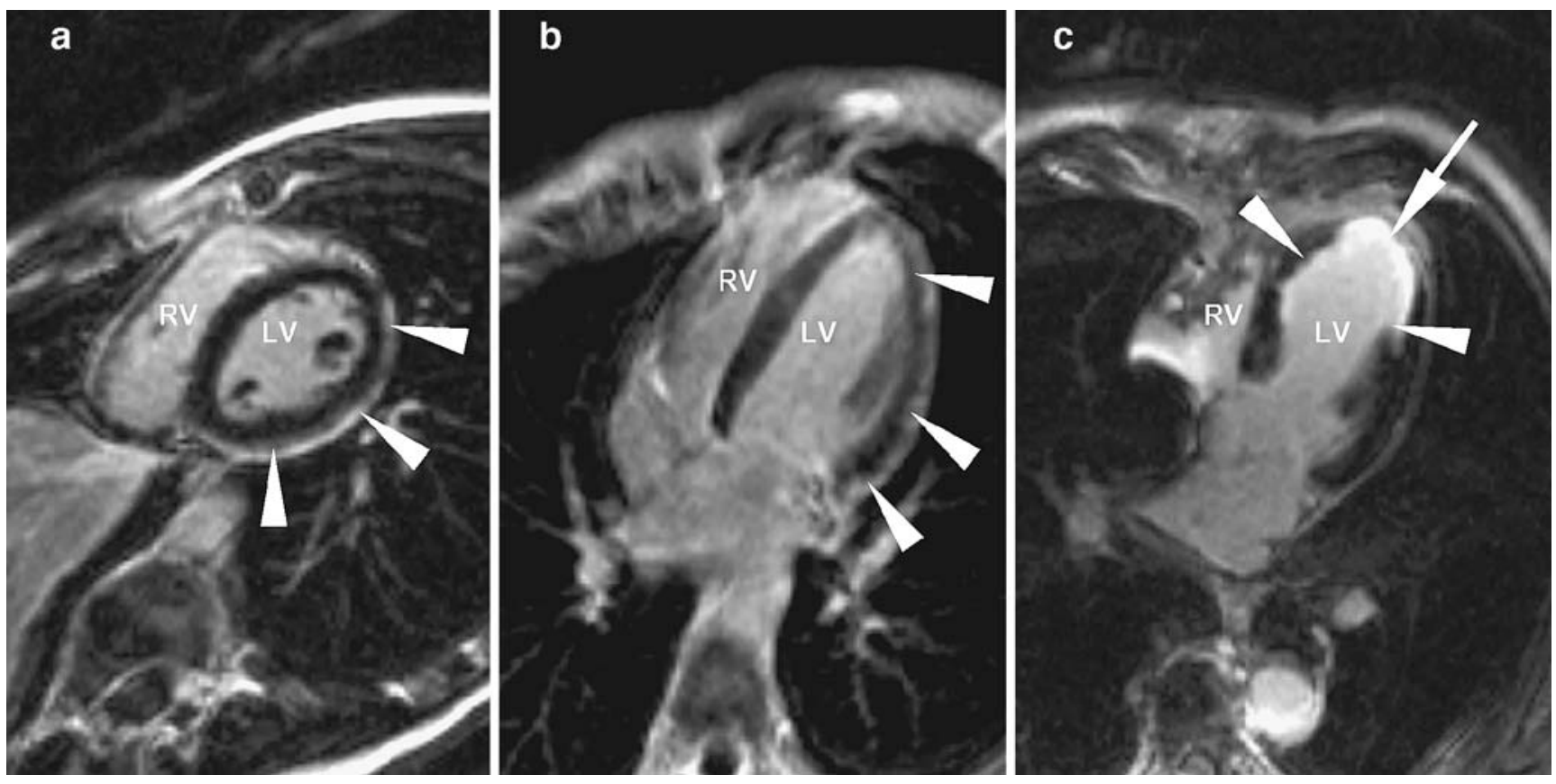

Fig. 1 Late contrast enhanced CMR (3D segmented FLASH sequence with inversion-recovery preparation [TR: 700, TE:1.53, TI: 250 , slice thickness $4 \mathrm{~mm}$ ) ) acquired 10 minutes after injection of $0.2 \mathrm{mmol} / \mathrm{kg}$ gadolinium in myocarditis (a and $\mathbf{b}$ ) and in myocardial infarction (c). $\mathbf{a}$ and $\mathbf{b}$ Short axis view and four-chamber view, respectively, showing enhancement of the subepicardial area in the inferior-lateral wall of the LV (arrowheads) typical of myocarditis. c Four-chamber view showing subendocardial enhancement in the lateral and septal wall of LV (arrowheads) and transmural enhancement with narrowing of the apical wall of the LV (arrow). LV: left ventricle; RV: right ventricle presence of edema, inflammation and necrosis in myocardial tissue. Recently, the necessity of endomyocardial biopsy has been challenged as late gadolinium enhanced CMR allows visualization of small myocardial injuries. Due to the different physiopathological processes resulting in myocardial lesions in both myocarditis and myocardial infarction, regions of contrast enhancement in late gadolinium enhanced CMR differ. In myocarditis, a patchy distribution originating primarily from the epicardium, most frequently located in the lateral free wall, is typically seen (Fig. 1a,b). In myocardial infarction, a focal subendocardial-transmural enhancement corresponding to a coronary artery territory is seen [3] (Fig. 1c).

Adult studies have demonstrated the appropriateness of delayed contrast enhanced CMR in distinguishing myocarditis from myocardial infarction [2, 3]. It appears to be the most promising technique for diagnosing myocardial inflammation and myocyte injury [4]. CMR will certainly become a standard diagnostic technique in patients with new onset of non-ischemic cardiac diseases $[5,6]$.

To date, no such studies have been performed in children. However, late contrast enhanced CMR is a safe, noninvasive diagnostic tool and might also be recommended in the pediatric population to distinguish myocarditis from myocardial infarction. Other diseases inducing nonischemic myocardial injury in children such as hypertrophic cardiomyopathies and other cardiomyopathies (i.e., Duchenne, Becker) may also benefit from this technique.

\section{References}

1. Cotran RS, Kumar V, Robbins SL (1994) Robbins pathologic basis of disease. Saunders, Philadelphia, pp 562-564

2. Hendel RC, Patel MR, Kramer CM, Poon M (2006) ACCF/ACR/ SCCT/SCMR/ASNC/NASCI/SCAI/SIR 2006 appropriateness criteria for cardiac computed tomography and cardiac magnetic resonance imaging. J Am Coll Cardiol 48:1475-1497

3. Laissy JP, Hyafil F, Feldman LJ, Juliard JM, Schouman-Claeys E, Steg PG, Faraggi M (2005) Differentiating acute myocardial infarction from myocarditis: diagnostic value of early- and delayed-perfusion cardiac MR imaging. Radiology 237:75-82

4. Magnani JW, Dec GW (2006) Myocarditis: current trends in diagnosis and treatment. Circulation 113:876-890

5. Mahrholdt H, Wagner A, Judd RM, Sechtem U, Kim RJ (2005) Delayed enhancement cardiovascular magnetic resonance assessment of non-ischaemic cardiomyopathies. Eur Heart J 26:14611474

6. Sechtem U, Mahrholdt H, Hager S, Vogelsberg H (2006) New noninvasive approaches for the diagnosis of cardiomyopathy: magnetic resonance imaging. Ernst Schering Res Found Workshop 55:261285 